\title{
A Case of Localized Fungal Pneumonia Caused by Rhodotorula mucilaginosa in an Immunocompetent Patient
}

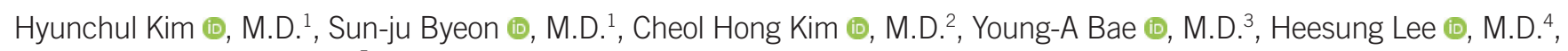
and Hyun Soo Kim (10, M.D. ${ }^{5}$

Departments of ${ }^{1}$ Pathology, ${ }^{2}$ Internal Medicine, ${ }^{3}$ Radiology, ${ }^{4}$ Thoracic Surgery, and ${ }^{5}$ Laboratory Medicine, Hallym University Dongtan Sacred Heart Hospital, Hallym University College of Medicine, Hwaseong, Korea

Dear Editor,

Rhodotorula species, belonging to the family Sporidiobolaceae, are yeasts that are present in the environment and can cause opportunistic infections in immunocompromised patients [1, 2]. Among Rhodotorula species, only $R$. mucilaginosa, $R$. glutinis, and $R$. minuta have been reported to cause human infections $[1,2]$. Rhodotorula sp. are mostly isolated from the blood of immunocompromised patients, where they cause fungemia or catheter-related infections. Only one case of pneumonia caused by $R$. mucilaginosa has been reported to date in an immunocompromised patient [3]. We report the first case of fungal pneumonia caused by $R$. mucilaginosa in an immunocompetent patient. The infection was confirmed by fungal ribosomal RNA (rRNA) sequencing using wedge-resected lung tissue.

This case report was approved by the Institutional Review Board of Hallym University Dongtan Sacred Heart Hospital, Hwaseong, Korea (IRB No. 2020-03-017). A waiver of consent was granted due to the retrospective nature of this study. A 52-year-old man visited our hospital in July 2019 because of a pulmonary nodule found incidentally during a health check-up at a local clinic in May 2019. The patient did not have any underlying disease and his complete blood count was within the normal range. A lobu- lated nodular lesion measuring $3.5 \times 1.6 \mathrm{~cm}$ was observed in the left lower lobe on radiological examination (computed tomography (CT); Fig. 1A). Serological screening tests for parasites (Clonorchis, Paragonimus, Cysticercus, Sparganum, and Toxocara) and the Aspergillus antigen (Galactomannan) test yielded negative results. Histopathological evaluation of a percutaneous needle biopsy revealed scattered, brown, oval, yeast spores (5-7 $\mu \mathrm{m})$ and chronic granulomatous inflammation, leading us to suspect fungal infection (Fig. 1B) [4]. Fungal culture using needle biopsy tissue was not performed at that time.

Empirical antifungal therapy with fluconazole $400 \mathrm{mg} /$ day was initiated and continued for three months (Aug 14-Nov 23, 2019), because cryptococcal infection was suspected based on the histopathological findings. During antifungal treatment, $\mathrm{CT}$ revealed a slight decrease in nodule size $(2.6 \times 1.6 \mathrm{~cm})$, which increased (to $3.1 \times 1.9 \mathrm{~cm}$ ) three months after discontinuation of the antifungal therapy. Wedge resection of the lung lesion was performed for definite diagnosis and treatment on March 5, 2020, and the fresh specimen was partitioned and sent for histopathological evaluation, fungal culture, and fungal rRNA sequencing. The histopathological features of the wedge-resected lung tissue were similar to those of the previous needle biopsy specimen.
Received: March 26, 2020

Revision received: April 21, 2020

Accepted: July 20, 2020

Corresponding author: Hyun Soo Kim, M.D.

Department of Laboratory Medicine, Hallym University Dongtan Sacred Heart Hospital, College of Medicine, Hallym University, 7 Keunjaebong-gil, Hwaseong 18450, Korea

Tel: +82-31-8086-2775, Fax: +82-31-8086-2789

E-mail: hskim0901@empas.com 


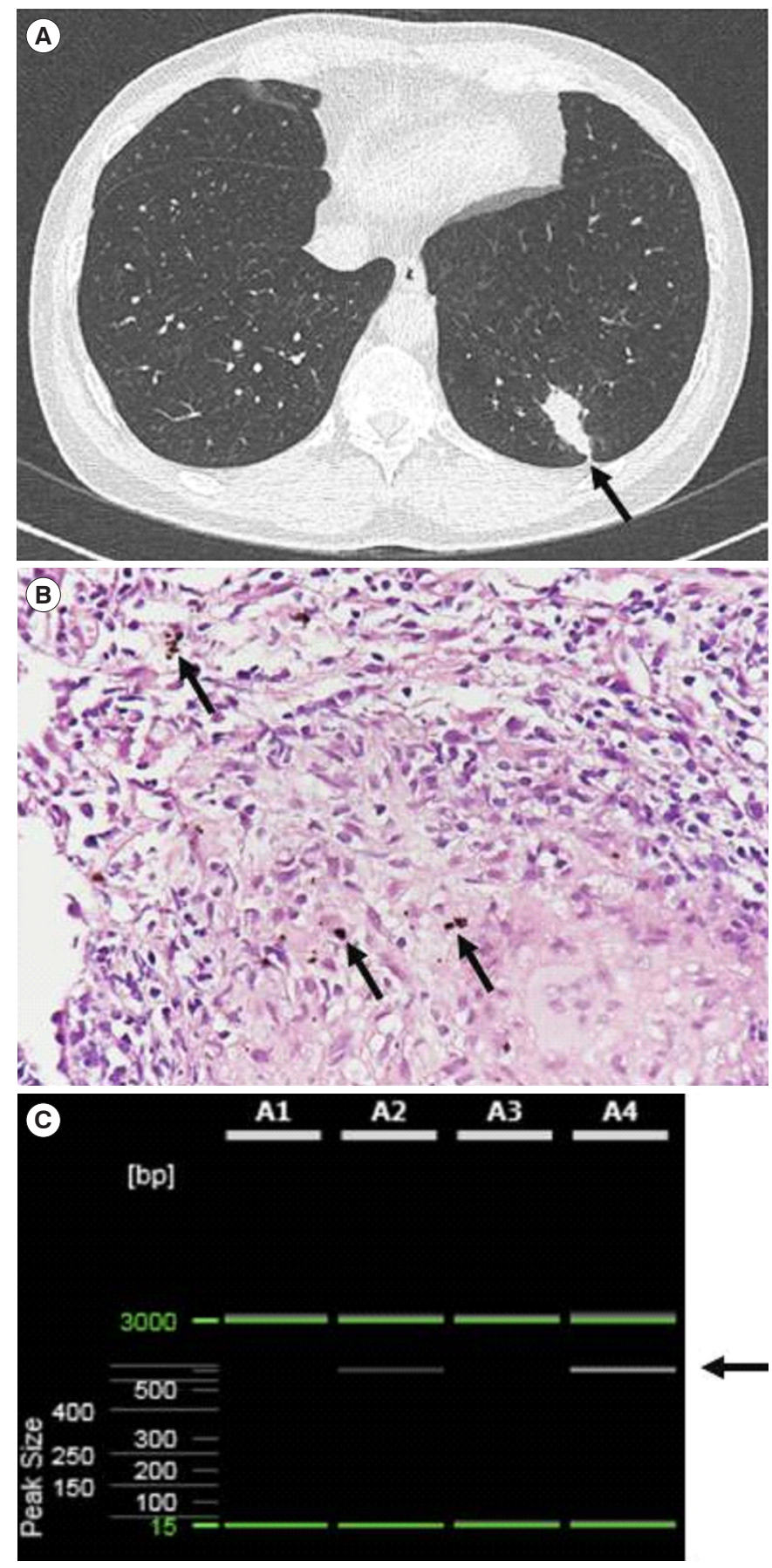

Fig. 1. Radiologic, histopathological, and PCR findings regarding Rhodotorula pneumonia for the case presented. (A) Chest CT scan shows a nodular lesion (arrow) in the left lower lobe. (B) The lung biopsy section shows scattered, brown, oval, yeast spores (arrows) and chronic granulomatous inflammation (Hematoxylin-eosin stain, $\times 400$ ). (C) Capillary electrophoresis of PCR products shows no amplicon for the fungal ITS1 region (lanes A1 and A3) and distinct amplicons for the fungal D1/D2 region of the large subunit rRNA (lanes A2 and A4).

Abbreviations: CT, computed tomography; rRNA, ribosomal RNA; ITS1, internal transcribed spacer 1 .
Fungal culture of the resected lung tissue showed no growth, probably due to the antifungal treatment.

DNA extracted from the wedge-resected lung tissue was used for PCR amplification and sequencing of the fungal internal transcribed spacer 1 (ITS1) region and the D1/D2 region of the large subunit rRNA gene (Fig. 1C) [5]. The ITS1 region was not amplified; however, the D1/D2 region of the large subunit rRNA gene showed $100 \%$ (560 bps /560 bps) sequence identity with the corresponding region in $R$. mucilaginosa based on a basic local alignment search tool using the sequence obtained (https: //blast.ncbi.nlm.nih.gov/).

As $R$. mucilaginosa pneumonia has never been reported in immunocompetent patients, it was extremely difficult to draw the differential diagnosis of pulmonary nodules. We thought that cryptococcal infection was the most likely type of fungal infection and began antifungal treatment. $R$. mucilaginosa pneumonia was diagnosed based on the correlation between histopathological findings and fungal rRNA sequencing data. Rhodotorula contamination from the environment could be ruled out, as similar histopathological findings regarding fungal morphology in granulomatous regions were observed in two biopsy sections taken six months apart. This patient was discharged after wedge biopsy, and the solitary pulmonary nodule completely disappeared on follow-up chest X-ray, one month later.

This case is unique compared with the previously-reported Rhodotorula infection cases from Korea, which involved either immunocompromised patients or patients undergoing invasive procedures (i.e., central venous catheterization and continuous peritoneal dialysis) [6-8], in that our patient was immunocompetent, without ongoing invasive procedures.

In summary, we report the first case of $R$. mucilaginosa pneumonia in an immunocompetent patient. The diagnosis was made based on combined histopathological and fungal rRNA sequencing findings in wedge-resected lung tissue. $R$. mucilaginosa pneumonia infection should be considered when conducting differential diagnosis for pulmonary nodules in immunocompetent patients.

\section{ACKNOWLEDGEMENTS}

We are grateful to Ju Un Park for the excellent technical assistance.

\section{AUTHOR CONTRIBUTIONS}

HSK designed and performed the study, obtained ethical ap- 
proval from IRB, and wrote the manuscript; HK collected the data and wrote the manuscript; $\mathrm{CHK}$ and $\mathrm{HL}$ diagnosed and treated the patient, provided clinical information, and edited the manuscript; YB, SB, HK, and HSK contributed to the diagnosis of Rhodotorula infection. All authors read and approved the final manuscript.

\section{CONFLICTS OF INTEREST}

None declared.

\section{RESEARCH FUNDING}

None declared.

\section{ORCID}

Hyunchul Kim

Sun-ju Byeon

Cheol Hong Kim

Young-A Bae

Heesung Lee
Hyun Soo Kim

https://orcid.org/0000-0002-7026-6715

\section{REFERENCES}

1. Wirth F and Goldani LZ. Epidemiology of Rhodotorula: an emerging pathogen. Interdiscip Perspect Infect Dis 2012;2012:465717.

2. Tuon FF and Costa SF. Rhodotorula infection. A systematic review of 128 cases from literature. Rev Iberoam Micol 2008;25:135-40.

3. Fischer J, Hamacher L, Fries J, Hallek M, Cornely OA, Kochanek M et al. Rhodotorula mucilaginosa as a cause of recurrent pulmonary infection and liver infiltration in a patient with CLL. Ann Hematol 2016;95: 1569-70.

4. Guarner J and Brandt ME. Histopathologic diagnosis of fungal infections in the 21st century. Clin Microbiol Rev 2011;24:247-80.

5. CLSI. Interpretive criteria for identification of bacteria and fungi by targeted DNA sequencing. 2nd ed. CLSI MM18. Wayne, PA: Clinical and Laboratory Standards Institute. 2018.

6. Bae TW, Lee J, Cho YG, Kim DS, Choi SI, Lee HS. Sepsis due to Rhodotorula mucilaginosa in a patient with advanced non-small cell lung cancer. Lab Med Online 2016;6:102-5.

7. Hong HK, Kim YM, Kim SD, Bae CW, Lee HJ. A case of Rhodotorula mucilaginosa peritonitis undergoing continuous ambulatory peritoneal dialysis in a neonate with acute renal failure. Neonatal Med 2003;10: 94-8.

https://orcid.org/0000-0002-9599-4970

https://orcid.org/0000-0002-8815-800X

https://orcid.org/0000-0001-7719-0154

https://orcid.org/0000-0002-2615-8346 nosa fungemia in an immunocompetent host. Infect Chemother 2013; 45:339-42. 